\title{
Testing in silkworms provides preventative diabetes treatment
}
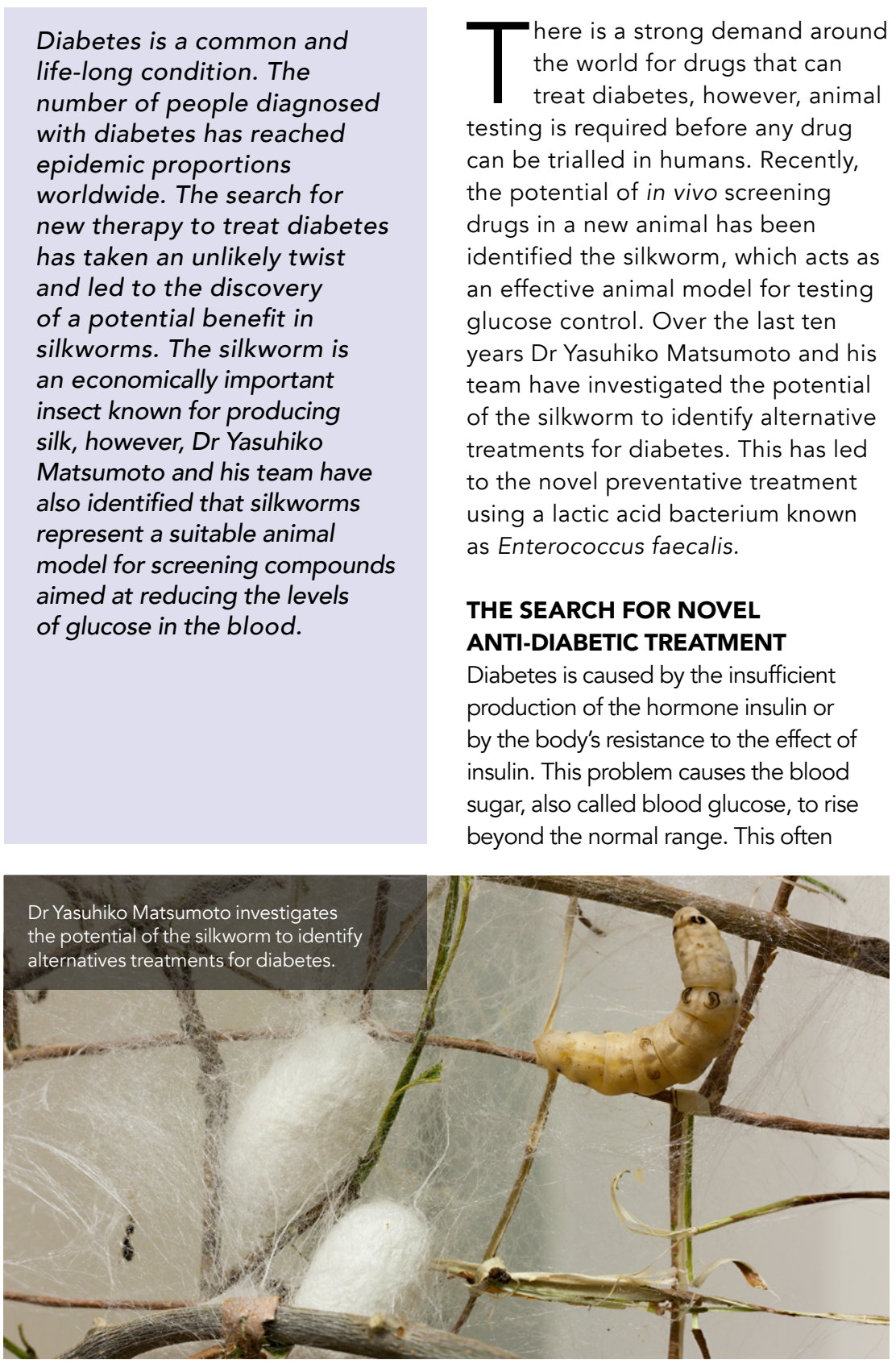

leads to a range of complications across the body, from frequent skin infections to complications occur due to the Arsange of nature of diabetes, therefore affecting and tissues. Glucose is an important energy source needed for all. the cells and organs of the body however like most things, it is best in moderation.

Type 1 diabetes is an auto-immune disease that is caused by the body's inability to produce insulin. Type 2 diabetes is the most common of the two subtypes usually caused by genetics and lifestyle and presents later in life. According to the World Health Organization, the number of people globally with diabetes has risen from 2014. The number of 422 million in with the nundition is expeple diagnosed Therefore, the need for diabetic drugs is major necessity However testing these drugs comes with its own challenges.

As blood glucose control requires hormonal control throughout the body. it is necessary to test the efficacy of newly identified anti-diabetic drugs in animal-models. Testing anti-diabetic drugs in diabetic mammals (such as mice and rats) has played a crucial role to the advancement of our knowledge and understanding of diabetes. However, the sacrifice of large numbers of mammals raises ethica concerns and is a very expensive method of testing.

THE SILKWORM MODEL

The silkworm, otherwise known by its

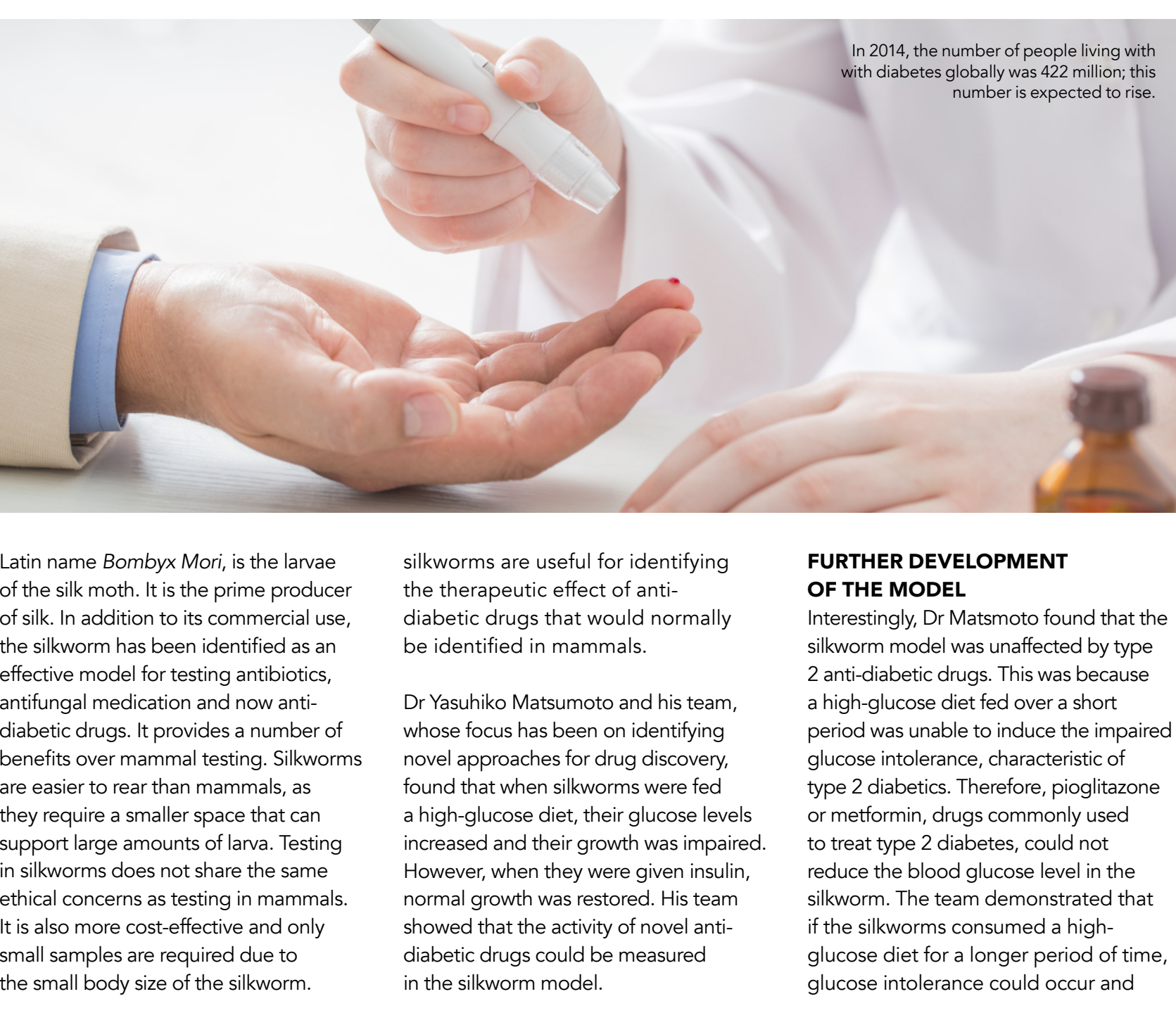

Surprisingly, the silkworm shares similar drug toxicity and basic pharmacokinetics (how the body treats a drug) with mammals. This makes the silkworm a

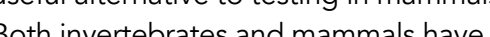
heir blood glucose levels regulated by the insulin-signalling pathway. However, in the silkworm, bombyxin is the name given to the insulin-like peptide that acts like insulin found in humans. Dr Matsumoto and his team previously found that by feeding silkworms a diet high in glucose, hyperglycaemia could be induced.

The total amount of sugar in the haemolymph, which is analogous to blood in mammals, was taken up by the fat body of the invertebrate which is also similar to the effects that occur is team also ide tified those tisure. His team also identified that human is
The hyperglycaemic silkworm model may be highly useful for quickly evaluating the therapeutic effects of anti-diabetic drugs.

In vivo infection models with inverteble animals and mice

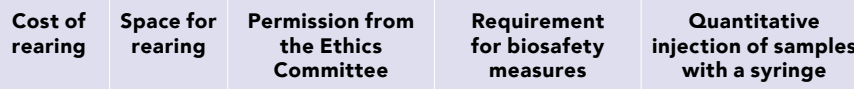

\begin{tabular}{|c|c|c|c|c|c|}
\hline $\begin{array}{l}\text { Silkwom (larva) } \\
\text { [Bombyk mori] }\end{array}$ & Low & Small & Not necessary & Low & Easy \\
\hline $\begin{array}{l}\text { Fruit fly (adulit) } \\
\text { [Drosophila] }\end{array}$ & Low & Small & Not necessary & High & Difficult \\
\hline $\begin{array}{l}\text { Nematode } \\
\text { |Ceanorithabitits } \\
\text { elegans| }\end{array}$ & Low & Small & Not necessary & Low & Difficult \\
\hline $\begin{array}{l}\text { Greater wax moth } \\
\text { (lavral Gillaria } \\
\text { mellonella] }\end{array}$ & Low & Small & Not necessary & Low & Easy \\
\hline $\begin{array}{l}\text { Mouse [Mus } \\
\text { musculus] }\end{array}$ & High & Large & Necessary & High & Easy \\
\hline
\end{tabular}




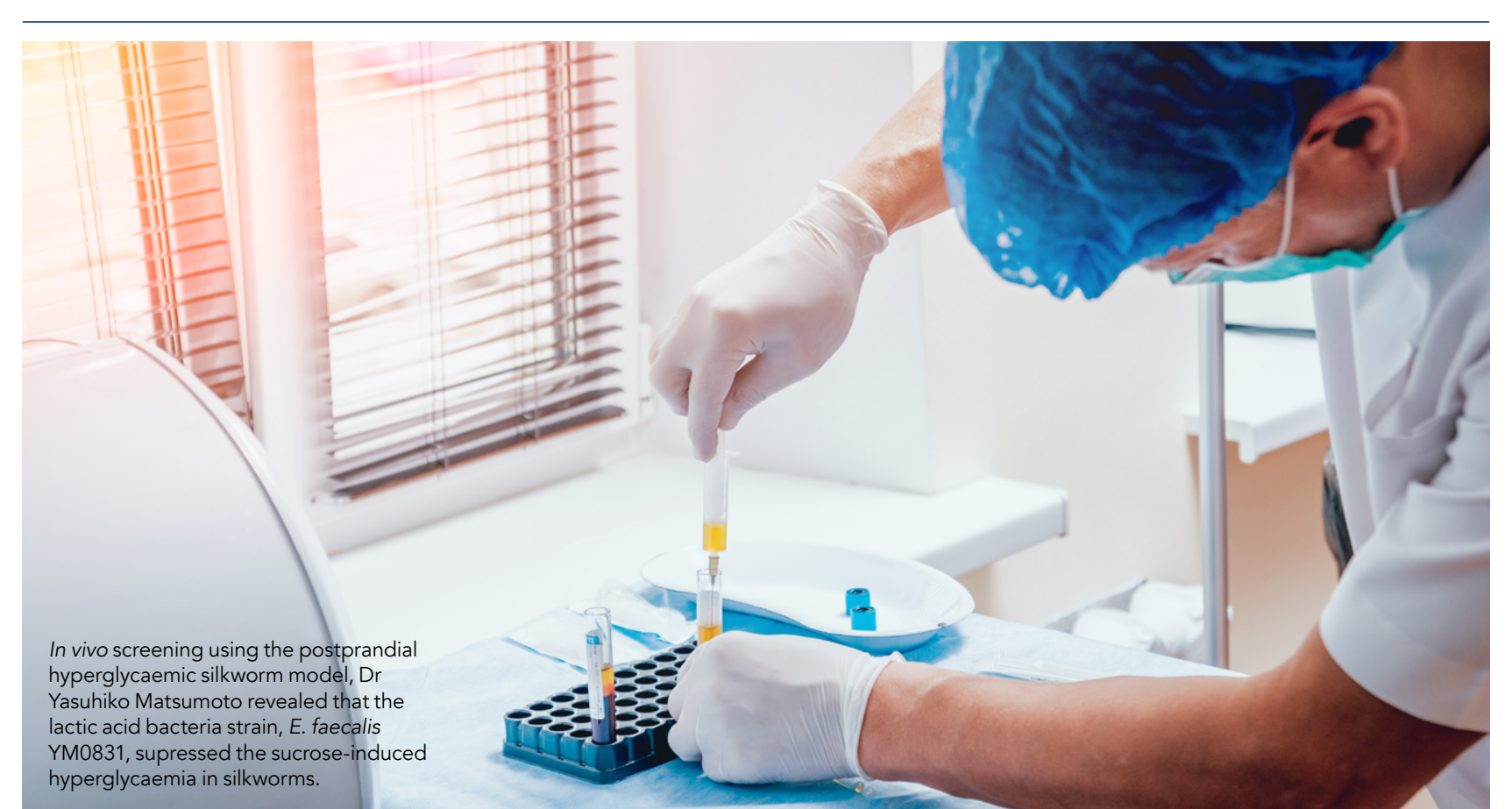

thus provided a type 2 diabetic animal model. is useful for evaluation of the type 2 anti-diabetic drugs, metformin and pioglitazone.

Sucrose is a common sugar, frequently added to sweeten food. The sucroseinduced postprandial hyperglycaen is linked to the onset of diabetes.
When sucrose undergoes digestion

strain, a type of lactic acid bacteria, could suppress the increase of blood glucose a similar effect in humans is lacking.

By large-scale in vivo screening using the postprandial hyperglycaemic silkworm model, Dr Yasuhiko Matsumoto revealed that the lactic acid bacteria strain, Enterococcus faecalis YM0831, supressed the sucrose-induced

modified bacterium also reduced the inhibitory activity of the bacterium's ability to suppress hyperglycaemia inhibitory action of E. faecalis YMO831 in the intestinal cell directly affects hyperglycaemia in host animals.

Using the silkworm model, this study found that a preventative approach for treating diabetes using the $E$. faecalis

in the body, it

is broken down

to fructose and

glucose by an

enzyme known

as $\alpha$-glycosidase.

allows sugars to

be absorbed in

he intestinal tratta

E. faecalis YM0831 inhibits intestinal glucose transport and suppresses sucrose-induced hyperglycaemia

in humans.

DrYasuhiko

Matsumoto found

use of $E$ faecals

in humans.

(1) . added to food to prevent glucose absorption, could suppress the increase in blood glucose by intake of sucrose.

\section{DISCOVERING ENTEROCOCCUS} AECALIS YM0831 USING

SILKWORMS

To identify treatments that could prevent hyperglycaemia, the team focused on bacterial strains that produced lactic acid as a by-product. Lactic acid bacteria ace used commercilly to ferment food e.g. identified that Lactobacillius rhamnosus hyperglycaemia in silkworms. E. faecalis
YM0831 also inhibited the uptake of YM0831 also inhibited the uptake of line otherwise known as Caco-2 and supressed hyperglycaemia caused by the ingestion of sucrose in humans. The findings indicate that a substance identified by silkworm model is effective to human health.

E. faecalis YM0831 was found to supress the increase of blood glucose in the silkworm. Dr Matsumoto and his team used a lactic acid bacterium that had been genetically modified to have reduced inhibitory action for glucose uptake in the intestinal cell line.
They found that the same genetically YMO831, functiona

hyperglycaemia in humans.

The silkworm model has demonstrated the potential of using E. faecalis YM083 for preventing hyperglycaemia in diabetic patients as a form of preventative viable and efficient in vivo screening model that could potentially replace the common use of diabetic mammals in anti-diabetic drug discovery. Notably, this model has been shown to be effective in other major therapeutic areas such as identifyng anti-fungals, antivirals, and screening tool for many oth mpontant reatment. Their research provides a antibiotics. With further research, the

\section{Behind the Research}

Af A A A

\section{Yasuhiko Matsumoto}

E: ymatsumoto@my-pharm.ac.jp T: +81-42-495-8745 W: https://researchmap.jp/Matsumon1980/

\section{Research Objectives}

Dr Matsumoto focuses on the development of nove approaches for drug discovery and preventiven using invertebrate animal models. In particular, he aims to eveal molecular mechanisms of infectious diseases and life-related diseases through molecular biology, chemical biology, and using novel invertebrate animal models.

\section{Detail}

Yasuhiko Matsumoto Ph.D.

Department of Microbiology

2-522-1 Noshio, Kiyose, Tokyo

204-8588 Japan

Bio

Yasuhiko Matsumoto is an Associate Professor of Meij Pharmaceutical University. He completed his Ph.D.

at the Graduate School of Pharmaceutical Sciences,

The University of Tokyo. He worked as Assistant Professor, The University of Tokyo and as Lecturer at Teikyo

Fundin

SPS KAKENHI (JP15H05783, JP17K08288) Suzuken Memorial Foundation (15-081)

Yakult Bio-Science Foundation

Supporting Industry Program by Ministry of Econom Trade and Industry

Collaborators

Dr Kazuhisa Sekimizu

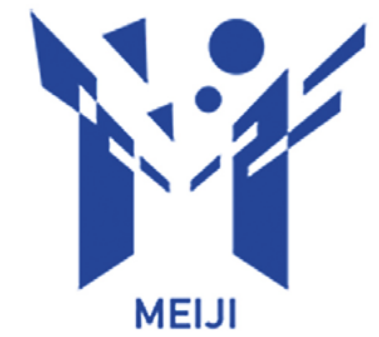

PHARMACEUTICAL UNIVERSITY
References

Matsumoto, Y. et al., (2011). An Invertebrate Hyperglycemic Model for the ldentification of Anti-Diabetic Drugs. Plos One, 6(3). https://doi.org/10.1371/journal.pone.0018292

Matsumoto, Y. et al., (2015). Diabetic silkworms for evaluation of therapeutically effective drugs against type II diabetes. Reports, 5.10722 doi:10.1038/srep10722

Matsumoto, Y. et al., (2016). An in vivo invertebrate evaluation system for identifying substances that suppress sucrose-induced postprandial hyperglycemia. Scientific Reports, 19;6:26354. doi:10.1038/srep26354

Matsumoto, Y. et al., (2019). Enterococcus faecalis YM083 suppresses sucrose-induced hyperglycemia in a silkworm model and in humans. Communication Biology, 157 (2019). doi:10.1038/s42003-019-0407-5

Matsumoto, Y. et al., (2019). Silkworm as an experimental animal for research on fungal infections. Microbiology and Immunology, 63(2): 41-50. doi:10.1111/1348-0421

\section{Personal Response}

What are some of the potential benefits that may to create functional food for diabetic patients?

U E. faecalis YM0831 is a lactic acid bacterium therefore it is useful for producing yogurt, a functional intake. We have demonstrated that yogurt produced by the E. faecalis YM0831 suppressed sucrose-induced hyperglycaemia in humans. Daily intake of the yogurt and results in prevention of onset of diabetes.

Could the commercial use of E. faecalis YM0831 to Coster gestational diabetes as wel?

II Yes. Daily intake of the yogurt produced by the E. faecalis YM0831 might be effective to prevent postprandial hyperglycaemia. 